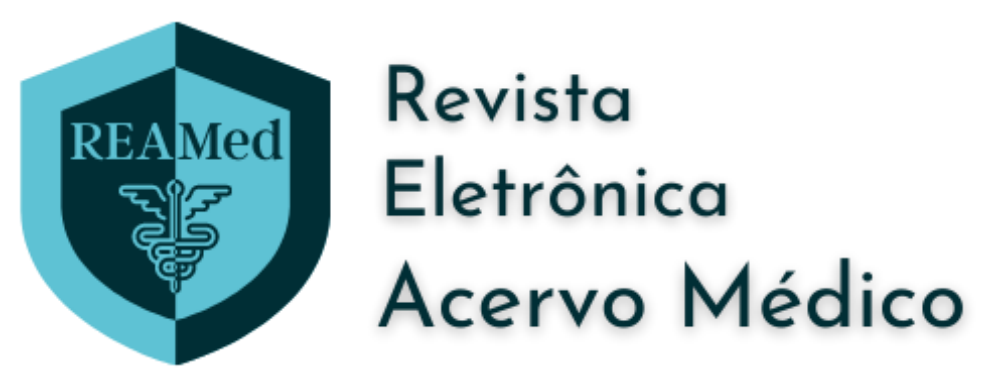

\title{
Análise do perfil de pacientes vítimas de trauma vesical em um hospital público de Alagoas
}

Analysis of victims profile of patients due to bladder trauma in a public hospital in Alagoas

Análisis del perfil de víctima de pacientes con trauma de vejiga urinaria en un hospital público de Alagoas

Reginaldo Melo Filho ${ }^{1 *}$, Maria Clara Motta Barbosa Valente ${ }^{1}$, Thiago Henrique Aquino Tenório ${ }^{1}$, Maria Helena Leitão Gomes¹, Anna Caroline Moreira Tenório", Carlos Eugênio Lira Tenório.

\section{RESUMO}

Objetivo: Analisar as características e incidência do trauma vesical durante cinco anos em um hospital geral do estado de Alagoas. Métodos: Estudo quantitativo, analítico, observacional e transversal, foram utilizados métodos de estatística descritiva para análise das variáveis. Resultados: Foram encontradas lesões vesicais em 63 pacientes, obteve-se análise dos prontuários nos anos de 2010 a 2015. Média de idade de 26,2 anos, com extremos entre 15 e 56 e desvio padrão de 9,85 (intervalo de confiança de $95 \%$ ). A maioria era (76,2\%) do sexo masculino. O trauma penetrante foi responsável por $65,07 \%$ dos casos, sendo em sua maioria $(97,56 \%)$ decorrente de lesão por arma de fogo. O método diagnóstico foi predominantemente intraoperatório $(82,53 \%)$. As lesões mais frequentes foram de cúpula vesical $(23,8 \%)$, parede anterior e posterior $(11,11 \%)$. O tratamento de escolha foi cirúrgico em $88,89 \%$ e conservador nos demais. A taxa de mortalidade global no trauma vesical foi de $7,93 \%$ a sobrevida média de $88,87 \%$. Conclusão: Os dados encontrados são, em sua grande parte, condizentes com a literatura mundial para o trauma vesical, diferindo quanto a etiologia, método diagnóstico e a terapêutica adotada. Tais distinções, podem ser justificadas pelas características locais e do hospital estudado.

Palavras-chave: Bexiga urinária, Pelve, Ferimentos e lesões, Traumatismo múltiplo.

\section{ABSTRACT}

Objective: To analyze the characteristics and incidence of bladder trauma over a five-year period in a general hospital in the state of Alagoas. Methods: Quantitative, analytical, observational and cross-sectional study, descriptive statistical methods were used to analyze the variables. Results: Bladder lesions were found in 63 patients, analysis of medical records was obtained from 2010 to 2015. Mean age of 26.2 years, with extremes between 15 and 56 and standard deviation of 9.85 (confidence interval of $95 \%$ ). Most were $(76.2 \%)$ male.

1 Universidade Estadual de Ciências da Saúde de Alagoas (UNCISAL), Maceió - AL.

*E-mail: reginaldomelofi@gmail.com 
Penetrating trauma was responsible for $65.07 \%$ of the cases, mostly $(97.56 \%)$ resulting from firearm injuries. The diagnostic method was predominantly intraoperative $(82.53 \%)$. The most frequent injuries were of the bladder dome $(23.8 \%)$, anterior and posterior wall $(11.11 \%)$. The treatment of choice was surgical in $88.89 \%$ and conservative in the others. The overall mortality rate forbladder trauma was $7.93 \%$ and the median survival $88.87 \%$. Conclusion: The data found are, for the most part, consistent with the world literature on bladder trauma, differing in terms of etiology, diagnostic method and adopted treatment. Such distinctions can be justified by the local characteristics and the studied hospital.

Key words: Urinary bladder, Pelvis, Wounds and injuries, Multiple trauma.

\section{RESUMEN}

Objetivo: Analizar las características e incidencia del traumatismo vesical durante un período de cinco años en un hospital general del estado de Alagoas. Métodos: Estudio cuantitativo, analítico, observacional y transversal, se utilizaron métodos estadísticos descriptivos para el análisis de las variables. Resultados: Se encontraron lesiones vesicales en 63 pacientes, se obtuvo análisis de historias clínicas de 2010 a 2015. Edad media de 26,2 años, con extremos entre 15 y 56 y desviación estándar de 9,85 (intervalo de confianza del $95 \%)$. La mayoría eran $(76,2 \%)$ hombres. El traumatismo penetrante fue responsable del $65,07 \%$ de los casos, en su mayoría $(97,56 \%)$ como consecuencia de lesiones por arma de fuego. El método diagnóstico fue predominantemente intraoperatorio $(82,53 \%)$. Las lesiones más frecuentes fueron de cúpula vesical $(23,8 \%)$, pared anterior y posterior (11,11\%). El tratamiento de elección fue quirúrgico en el $88,89 \%$ y conservador en los demás. La tasa de mortalidad global por traumatismo vesical fue del $7,93 \%$ y la supervivencia media del $88,87 \%$. Conclusión: Los datos encontrados son, en su mayor parte, consistentes con la literatura mundial sobre trauma vesical, difiriendo en cuanto a etiología, método diagnóstico y tratamiento adoptado. Tales distinciones pueden estar justificadas por las características locales y el hospital estudiado.

Palabras clave: Vejiga urinaria, Pelvis, Heridas y lesiones, Traumatismo múltiple.

\section{INTRODUÇÃO}

O trauma constitui um dos principais problemas de saúde pública no mundo, isso porque cerca de 60 milhões de pessoas sofrerão algum trauma ao longo de um ano. Além disso, representa a terceira principal causa de óbitos no mundo e a primeira entre jovens adultos. Nesse contexto, o trauma abdominal é um dos mais prevalentes. Dividido em trauma abdominal contuso e penetrante, apresenta um alto potencial lesivo, além de estar relacionado a altas taxas de morbi/mortalidade (BATISTA SEA, et al., 2006; NÁCUL MP, et al., 2005; LIMA SO et al., 2012; QUAGLIANO PV, et al., 2006).

Aproximadamente 3 a $10 \%$ dos indivíduos vítimas de trauma terão lesão no trato geniturinário e lesões de bexiga ocorrerão em cerca de $1,6 \%$ desses casos. Dentre os mecanismos de lesão podemos citar traumas abdominais penetrantes por arma de fogo ou arma branca, contusos e lesões iatrogênicas decorrentes de procedimentos cirúrgicos principalmente de procedimentos obstétricos. As colisões em acidentes automobilísticos são a causa mais comum de trauma contuso de bexiga, tendo sua importância aumentada principalmente quando o trauma acontece em concomitância com a lesão do anel pélvico. $O$ trauma geniturinário raramente é fatal, mas pode evoluir com uma série de sequelas para os pacientes af etados, com comprometimento funcional e perda da qualidade de vida (BARNARD J, et al., 2019; MAHAT Y, et al., 2019; LIMA SO, et al., 2012).

A bexiga é o órgão urológico mais afetado por lesões iatrogênicas, que ocorrem normalmente em procedimentos obstétricos ou ginecológicos, da cirurgia geral e procedimentos urológicos. Entre as causas mais comuns pode-se citar: parto cesariano, histerectomia (abdominal ou vaginal), herniorrafias, slings, cirurgias para tratamento de prolapsos vaginais e ressecções transuretrais de bexiga e próstata, tendo seu cuidado redobrado em tais procedimentos para que se evite tais iatrogênias (GOMEZ RG, et al., 2004; TONKIN JB, et al., 2011). 
Já no que se refere ao trauma, as lesões vesicais podem ser classificadas como extraperitoneais e intraperitoneais, as quais são de extrema importância dif erencia-las para que se possa oferecer um tratamento adequado. As lesões extraperitoneais tem uma maior incidência ( $55 \%$ das lesões de bexiga) e são quase sempre associadas a fraturas pélvicas. Essas são comumente causadas pela distorção do anel pélvico, atingindo, usualmente, a parede anterolateral da bexiga, próximo à base, em suas fixações faciais. Além disso, associam-se com lesões de uretra em $15 \%$ dos casos e com lesão intra-abdominal em outros órgãos em 44\% (MAHAT Y, et al., 2019; GOMEZ RG, et al., 2004; RODDER K, ET AL., 2005).

Já as lesões vesicais intraperitoneais ocorrem pelo rápido aumento da pressão intravesical secundária a um trauma contuso sobre o abdome inf erior ou pelve, sendo a cúpula da bexiga a área de maior fragilidade e consequentemente de maior incidência de lesões vesicais no trauma contuso. Estudos demonstram que este é trauma de bexiga mais comumente encontrado (GOMEZ RG, et al., 2004; LYNCH TH, et al., 2005).

Os sintomas decorrentes do trauma vesical baseiam-se na severidade das lesões, são sintomas apresentados: hematúria macroscópica, dor supra púbica, dificuldade de micção, entre outros. A associação com o trauma pélvico é de extrema importância, sendo necessária a investigação de lesões de trato geniturinário sempre que diante de traumas pélvicos (BARNARD J, et al., 2019).

Conforme a Associação Americana de Urologia (AUA), é recomendada a cistografia retrógrada como exame de escolha para a identificação lesões vesicais. No que se refere ao tratamento, lesões extraperitoneais exigem tratamento o conservador, que consiste em sondagem vesical de demora por dez dias associada a antibioticoterapia. Já lesões do colo vesical, presença de fragmentos ósseos na parede vesical e lesão retal associada necessitarão de uma exploração cirúrgica, mesmo na presença de uma lesão extraperitoneal isolada. Em lesões intraperitoneais se faz necessária a abordagem cirúrgica e reparo primário da lesão, pelo risco elevado de peritonite e sepse intra-abdominal (GOMEZ RG, et al., 2004; RODDER K, et al., 2005; LYNCH TH, et al., 2005).

A realidade no que se refere ao trauma vesical já se encontra descrita na literatura, no entanto, foi observada a escassez de estudos atuais que demonstrem achados epidemiológicos acerca do trauma de bexiga. Além disso, não foram encontrados estudos que demonstrassem tal realidade no estado de Alagoas. Diante disso, o presente estudo teve como objetivo conhecer o perfil de pacientes com lesão vesical atendidos em um hospital de trauma do estado de Alagoas.

\section{METÓDOS}

Este projeto de pesquisa foi aprovado pelo Comitê de Ética em Pesquisa da Universidade Estadual de Ciências da Saúde de Alagoas (UNCISAL), Maceió, AL sob número do Parecer: 1.170.057.

Trata-se de um estudo com abordagem quantitativa, analítica, observacional e transversal, guiado por revisão de prontuários de vítimas de trauma vesical atendidas em um hospital de trauma do estado de Alagoas no período de julho de 2010 a julho de 2015.

Para a definição do número de participantes, não foi realizado cálculo amostral, tendo sido incluídos todos os prontuários dos pacientes vítimas de trauma admitidos no período de julho de 2010 a julho de 2015 .

As variáveis analisadas foram: idade, sexo, mecanismo de trauma, grau das lesões vesicais, método diagnóstico, conduta utilizada no tratamento, lesões associadas, complicações, sinais, sintomas e óbitos. As lesões foram classificadas de acordo com a Associação Americana de Cirurgia do Trauma (AAST). Foram excluídos prontuários de pacientes previamente atendidos em outros serviços e os prontuários incompletos quanto as variáveis adotadas pelo estudo.

A análise foi realizada por meio da coleta de dados dos prontuários dos pacientes que foram armazenados em uma planilha eletrônica (Microsoft Excel® 2010. Redmond, WA, EUA). Após a tabulação de dados foram feitos os cálculos de estatística descritiva, dentre esses: médias, desvio-padrão, análise de frequência e percentuais dos dados analisados, os quais foram demonstrados por meio de tabelas. Os cálculos foram realizados com o auxílio do aplicativo estatístico BioEstat 5.9.8. 


\section{RESULTADOS}

Foram analisados um total de 728 prontuários de pacientes vítimas de trauma, 63 pacientes registravam lesão vesical, com uma média por mês de 1,03 casos. Destes, 48 eram do sexo masculino $(76,2 \%), 10$ do sexo feminino $(15,87 \%)$ e cinco fichas de admissão não constavam o sexo do paciente $(7,93 \%)$. A relação homens e mulheres foi de 4,8:1 e a média das idades dos pacientes acometidos de trauma vesical foi de 25,6 anos com extremos entre 15 e 56 anos.

O mecanismo do trauma mais prevalente foi o do tipo penetrante, em 41 pacientes $(65,07 \%)$, sendo o ferimento por projétil de arma de fogo o mais prevalente com 40 casos $(63,49 \%$ - percentual total, $97,56 \%$ percentual entre os abertos). Já os ferimentos por arma branca (FAB) foram responsáveis por um caso (1,59\% - percentual total, 2,44\% - percentual entre os abertos). O trauma não penetrante ou fechado ocorreu em 22 pacientes (34,92\%), e suas causas foram: nove quedas de altura (14,29\%), seis acidentes automobilísticos (9,52\%), três acidentes motociclísticos e quatro não foram relatados nos prontuários $(6,34 \%)$ (Tabela 1).

Tabela 1 - Mecanismo do trauma vesical penetrante e não penetrante.

\begin{tabular}{ccc}
\hline Penetrante & Número de pacientes & Porcentagem (\%) \\
\hline Arma de fogo & 40 & 97,56 \\
Arma branca & 1 & 2,44 \\
\hline Total & 41 & 100 \\
\hline Não penetrante & & \\
\hline Quedas de altura & 9 & 40,91 \\
Acidente automobilístico & 6 & 27,7 \\
Acidente motociclísticos & 3 & 13,64 \\
Não identificados & 4 & 18,18 \\
\hline Total & 16 & 100 \\
\hline
\end{tabular}

Fonte: Filho RM, et al., 2021.

Quanto a localização anatômica das lesões vesicais, a lesão de cúpula vesical foi a mais encontrada (25,39\%), seguida pelas lesões em parede anterior e posterior da bexiga, cada uma com $11,11 \%$ de incidência (Tabela 2). Observa-se ainda na Tabela 2, que 30,16\% não tiveram a localização dalesão identificada, lesões simultâneas ocorreram em parede anterior e posterior e em colo e base, em 3,17\% e 1,59\% dos casos, respectivamente.

Tabela 2 - Localização anatômica das lesões vesicais.

\begin{tabular}{ccc}
\hline Local lesão & Número de pacientes & Porcentagem (\%) \\
\hline Cúpula & 16 & 25,39 \\
Parede posterior & 7 & 11,11 \\
Parede anterior & 7 & 11,11 \\
Colo & 4 & 7,93 \\
Base & 3 & 4,77 \\
Parede lateral & 2 & 3,17 \\
Parede anterior e posterior & 02 & 3,17 \\
Colo e base & 01 & 1,59 \\
Não identificada & 21 & 30,16 \\
\hline Total & 63 & 100 \\
\hline
\end{tabular}

Fonte: Filho RM, et al., 2021.

As lesões em órgãos associadas ao trauma vesical foram encontradas em $71,43 \%$ dos pacientes, sendo o intestino grosso e delgado os órgãos mais acometidos (45,50\%) e a lesão retal a mais comum (12 casos), como demostrado na Tabela 3. A vesícula biliar foi o órgão menos atingido com apena um caso $(2,50 \%)$ e o 
compartimento anatômico mais lesado foi o abdome (48,14\%), seguido por pelve $(29,62 \%)$, tórax $(14,81 \%)$ e crânio $(7,40 \%)$.

Tabela 3 - órgãos associados ao trauma vesical.

\begin{tabular}{ccc}
\hline Órgão & Número de pacientes & Porcentagem (\%) \\
\hline Intestino grosso/delgado & 18 & 45,0 \\
Reto & 12 & 30,0 \\
Ureter & 4 & 10,0 \\
Vasos ilíacos & 3 & 7,50 \\
Fígado & 2 & 5,0 \\
Vesícula biliar & 1 & 2,5 \\
\hline Total & 40 & 100
\end{tabular}

Fonte: Filho RM, et al., 2021.

A dor abdominal foi o sintoma mais encontrado entre os pacientes estudados $(54,92 \%)$, seguido pelo sinal de hematúria macroscópica (25,35\%), irritação peritoneal (9,85\%), hematúria microscópica $(4,22 \%)$, hematoma perineal $(5,63 \%)$.

A lesão extraperitoneal foi a mais encontrada das lesões não penetrantes $(50,00 \%)$, seguida pelas lesõ es intraperitoneais $(27,27 \%)$, sendo a lesão mista a de menor incidência nos pacientes encontrados $(7,40 \%)$ (Tabela 4).

Tabela 4 - Classificação das lesões não penetrantes.

\begin{tabular}{ccc}
\hline Classificação & Número de pacientes & Porcentagem (\%) \\
\hline Extraperitoneal & 11 & 50,00 \\
Intraperitoneal & 6 & 29,62 \\
Contusão & 4 & 14,81 \\
Mista & 1 & 7,40 \\
\hline Total & 22 & 100 \\
\hline
\end{tabular}

Fonte: Filho RM, et al., 2021.

O diagnóstico de lesão vesical foi dado em sua expressiva maioria no intraoperatório (52 casos - 82,53\%) esse dado se justifica principalmente por se tratar de pacientes politraumatizados e pela maior prevalência de lesões por arma de fogo, necessitando de uma abordagem cirúrgica de emergência, seguido pelo método clínico $(9,52 \%)$ e de imagem como ultrassonográfico $(6,35 \%)$ e tomográfico $(1,6 \%)$, de acordo com a Tabela 5.

Tabela 5 - Método diagnóstico das lesões vesicais.

\begin{tabular}{ccc}
\hline Método & Número de pacientes & Porcentagem (\%) \\
\hline Intraoperatório & 52 & 82,53 \\
Clínico & 6 & 9,52 \\
Ultrassonográfico & 4 & 6,35 \\
Tomografia computadorizada & 1 & 1,60 \\
\hline Total & 27 & 100 \\
\hline
\end{tabular}

Fonte: Filho RM, et al., 2021.

O tratamento mais realizado foi o cirúrgico com rafia primária da lesão vesical em 56 pacientes $(88,89 \%)$. Já o tratamento clínico, com sonda vesical de demora, hidratação endovenosa, antibioticoterapia e analgesia, foi instituído em 11 casos (11,11\%). Não houve nenhum óbito relacionado diretamente ao trauma vesical, a 
taxa de mortalidade no estudo foi de $7,93 \%$, sendo a causa mais comum de óbito o choque hipovolêmico (60\%), seguido por sepse (40\%). Tendo assim, uma sobrevida média dos pacientes analisados de $88,88 \%$.

\section{DISCUSSÃO}

Este estudo foi realizado no período entre 2010 e 2015 e teve como objetivo principal descrever o perfil epidemiológico de pacientes com trauma de bexiga em um hospital geral do estado de Alagoas. O hospital selecionado é a referência estadual para casos de trauma, por isso apresenta um elevado fluxo de pacientes diariamente. Sabe-se que o trauma é um grande problema de saúde pública que afeta a população como um todo. Nesse aspecto, apesar de não ser um dos traumas mais frequentes, o trauma de bexiga ganha relevância, pois constitui uma das principais lesões do trato geniturinário. Além disso, é considerado um trauma grave devido a necessidade de internamento prolongado e reabilitação (KHADJIBAEV AM, et al., 2016).

A bexiga é um órgão pélvico extraperitoneal protegido por uma porção óssea que constitui o arco pubiano anterior, o qual se diferencia em uma porção fina ancorada ao assoalho pélvico e uma porção óssea que se movimente segundo o grau de distensão da bexiga tornando-se de acorco com seu nível urinário em intraperitoneal ou extraperitoneal. Devido a essa condição anatômica de proteção, o trauma vesical torna-se uma patologia com prevalência baixa e que, quando encontrada, se produz por traumatismo fechado grave ou traumatismo penetrante (LYNCH TH, et al., 2005).

No presente estudo, o trauma penetrante foi o mais prevalente $(63,49 \%)$, diferindo do trabalho de Barnard $\mathrm{J}$, et al. (2019), no qual o trauma contuso porcolisão automobilística foi o mais prevalente (45\%). Já em outros trabalhos, como o de Vega CV, et al. (2012) e Cury J e Guglielmetti GB (2010), observa-se uma maior prevalência de traumas contusos de bexiga. Isso reflete a realidade dos traumas no estado de Alagoas, onde traumas penetrantes são frequentes.

Neste estudo foi encontrada uma maior incidência em pacientes do sexo masculino $(76,2 \%)$ e que possuíam a média de idade de 26,2 anos. Tal achado é compatível com os achados epidemiológicos já descritos em outros estudos como o de Vega CV, et al. (2012), no qual $81,25 \%$ dos casos são do sexo masculino. As lesões associadas mais frequentes foram as lesões do trato gastrointestinal, seguidas por fraturas pélvicas. O diagnóstico dessas lesões ocorreu principalmente no intraoperatório $(82,53 \%)$, acreditase que isso se deve ao perfil dos pacientes atendidos neste hospital, os quais são, em sua maioria, pacientes politraumatizados graves e com suspeita de lesões em outros órgãos abdominais, sendo indicada na maioria das vezes a laparotomia exploradora.

Estudos demonstram que cerca de $3 \%$ dos pacientes vítimas de trauma pélvico terão lesões vesicais associadas. Neste trabalho observou-se que $29,62 \%$ dos pacientes com lesão de bexiga apresentavam trauma de pelve. Nesse aspecto, evidencia-se a importancia da investigação de lesões vesicais em pacientes com esse mecanismo de trauma (HERTZ AM, et al., 2020).

Conforme o descrito pela literatura, em traumas contusos, lesões de outras vísceras abdominais associadas são as mais frequentes, além desses pode-se citar fraturas de ossos longos, lesões do sistema nervoso central e lesões torácicas. Já em traumas penetrantes lesões retais estão associadas em $38 \%$ dos casos, o que leva a um pior prognóstico, pelo maior risco de complicações com aumento da taxa de infecção associada e sepse (MAHAT Y, et al., 2019).

Neste estudo, o segundo método diagnóstico mais frequente foi a tomografia computadorizada seguida pela ultrassonografia, fato que pode ser explicado pela facilidade de acesso a tais métodos diagnósticos no hospital estudado. O que difere de outros estudos como o de Kivlin D, et al. (2015), no qual o método diagnóstico de maior de maior prevalência foi a uretrocistografia. Além disso, observa-se atualmente o papel do Focused Assesment with Sonography in Trauma (FAST) como uma avaliação rápida de lesões intraabdominais (BAIN K, et al., 2018).

Já do ponto de vista de tratamento observou-se a maior prevalência de abordagem cirúrgica $(88,89 \%)$, fato que pode ser explicado, pois o trauma penetrante foi o mais prevalente, necessitando assim de 
abordagem cirúrgica imediata. Observa-se que a abordagem cirúrgica precoce de lesões intraperitoneais melhora o prognóstico desses pacientes. Visto que podem evoluir com injúria renal aguda e sepse, caso o tratamento seja retardado, com uma taxa de mortalidade superior a $20 \%$. Dessa forma, parâmetros como níveis de pressão arterial sistólica inferiores a $90 \mathrm{mmHg}$ e presença de trauma pélvico estão relacionados a piores prognósticos. Já pacientes estáveis hemodinamicamente e com bons valores na Escala de Coma de Glasgow apresentam melhor prognóstico (ELKBULI A, et al., 2019; PHILLIPS B, et al., 2017).

Em pacientes com ruptura extraperitoneal o manejo conservador deve ser adotado. Neste estudo $11,11 \%$ dos pacientes foram submetidos ao tratamento conservador. Nesse aspecto evidencia-se a necessidade dos cuidados relacionados e inf ecções de trato urinário relacionadas ao uso de sonda vesical de demora, alem da antibioticoretapia. No serviço estudado não foi observado um protocolo institucional que orientasse as medidas a serem realizadas para esses pacientes (ELKBULI A, et al., 2019).

Este estudo apresentou algumas limitações, por se tratar de um estudo transversal com revisão de prontuários muitas informações não se encontravam registradas, o que, além de dificultar a coleta, pode ter interferido no registro dos dados, visto que tais prontuários não foram contabilizados nesta análise. Além disso, estudos transversais de prevalência que caracterizem o perfil de pacientes vítimas de trauma de bexiga são escassos, com poucos artigos publicados nos últimos cinco anos, o que dificultou uma melhor análise comparativa entre a amostra estudada e a literatura disponível, reiterando a importância deste estudo. Além disso, reforça-se a necessidade de fortalecimento de protocolos para o diagnóstico de tais lesões, em especial em traumas abdominais e pélvicos. Pois se sabe que lesões do trato geniturinário devem ser pesquisadas em pacientes vítimas de trauma pélvico ou que apresentem hematúria importante.

\section{CONCLUSÃO}

Neste estudo observou-se que o trauma penetrante foi o mais prevalente, em sua maioria decorrente de lesões por arma de fogo. Já entre os contusos, as quedas de altura foram a principal causa de lesões de bexiga. Quanto a localização das lesões, as não identificadas foram as mais frequentes, seguido de lesões de cúpula vesical. A lesão associada mais frequente foi a de intestino grosso e delgado. Na maioria dos casos o diagnóstico se deu no intraoperatório. O tratamento cirúrgico foi o mais realizado, além disso, a taxa de mortalidade no estudo foi de $7,93 \%$. Diante disso, observa-se que a caracterização epidemiológica do perfil de tais pacientes é de extrema valia de modo a permitir ações preventivas e terapêuticas a serem implantadas pelo sistema de saúde do Estado do hospital estudado.

\section{REFERÊNCIAS}

1. BAIN K, et al. FAST examination diagnosing bladder rupture following blunt pelvic trauma. British Medical Journal Case Report, 2018; bcr2017223933.

2. BARNARD J, et al. Traumatic Bladder Ruptures: A Ten-Year Review at a Level 1 Trauma Center. Advances in Urology, 2019;2614586.

3. BATISTA SEA, et al. Análise comparativa entre os mecanismos de trauma, as lesões e o perfil de gravidade das vítimas, em Catanduva-SP. Rev. Col. Bras. Cir., 2006;33(1): 6-10.

4. CURY J, GUGLIELMETTI GB. Trauma Genitourinário. In: NARDOZZA AJ, et al. Urologia Fundamental - Sociedade Brasileira de Urologia. São Paulo: Planark, 2010;302-304.

5. ELKBULIA, et al. Management of blunt intraperitoneal bladder rupture: case report and literature review. International Journal of Surgery Case Reports, 2019;55: 160-163.

6. GOMEZ RG, et al. Consensus statement on bladder injuries. BJU Int., 2004;94(1): 27-32.

7. HERTZ AM, et al. Identifying Bladder Rupture Following Traumatic Rupture Following Traumatic Pelvic Fracture: A Machine Learning Approach. Injury, 2020;51(2): 334-339.

8. KIVLIN D, et al. A Case Series of Spontaneous Rupture of the Urinary Bladder. Curr. Urol., 2015; 8(1):53-56.

9. KHADJIBAEV AM, et al. Bladder trauma: a 7 year review. Urol. Nephrol. Open Access J., 2016;5(3): 00175.

10. LIMA SO, et al. Avaliação epidemiológica das vítimas de trauma abdominal submetidas ao tratamento cirúrgico. Rev. Col. Bras. Cir., 2012; 39(4):302-306.

11. LYNCH TH, et al. EAU guidelines on urological trauma. Eur. Urol., 2005; 47(1):1-15.

12. MAHAT Y, et al. A contemporary review of a adultbladder trauma. J. Inj. Violence Res., 2019; 11(2): $101-106$.

13. NÁCUL MP, et al. Videolaparoscopia no trauma abdominal contuso. Rev. Bras. Videocir., 2005;3(4): $196-207$. 
14. PEREIRA BMT, et al. Bladder injuries after external trauma:20 years experience report in a population-based crosssectional view. World J. Urol., 2013;31(4): 913-917.

15. PHILLIPS B, et al. Trauma to the bladder and ureter: a review of diagnosis, management, and prognosis. Eur. J. Trauma Emerg. Surg., 2017;43(6): 763-773.

16. QUAGLIANO PV, et al. Diagnosis of blunt bladder injury: A prospective comparative study of computed tomography cystography and conventional retrograde cystography. J. Trauma, 2006;61 (2): 410-421.

17. RODDER K, et al. Bladder injury diagnostics and treatment. Urologe A., 2005;44(8): $878-82$.

18. TONKIN JB, et al. Assessment and initial management of urologic trauma. Med. Clin. North Am., 2011; 95(1): 245251.

19. VEGA CV, et al. Experiencia del manejo del trauma vesical en Hospital de Urgencia Asistencia Pública. Revisión de cinco años, 2005-2009. Rev. ANACEM, 2012;6(2): 76-79.

20. XISHUANGS, et al. Comparing the safety and efficiency of conventional monopolar, plasmakinetic, and holmium laser transurethral resection of primary non-muscle invasive bladder cancer. J. Endourol., 2010; 24(1): 69-73.

21. WIRTH GJ, et al. Advances in the management of blunt traumatic bladder rupture: experience with 36 cases. BJU Int., 2010; 106(9): 1344-1349.

22. ZINMAN LN, VANNI AJ. Surgical management of urologic trauma and iatrogenic injuries. Surg. Clin. North Am., 2016; 96(3): 425-439. 